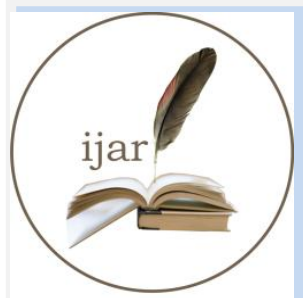

ISSN NO. $2320-5407$
Journal Homepage: -www.journalijar.com INTERNATIONAL JOURNAL OF ADVANCED RESEARCH (IJAR)

Article DOI: $10.21474 / \mathrm{IJAR} 01 / 1730$

DOI URL: http://dx.doi.org/10.21474/IJAR01/1730

\title{
A StrategicFramework forEradication ofChild Labor in Delhi: AStudy on ChildLabor in Dhabas of Delhi.
}

${ }^{1}$ Dr.Poonam Khurana, Associate Professor- Vivekananda Institute of Professional Studies, GGSIPU, Delhi

${ }^{2}$ Ms.Nidhi Sharma, Assistant Professor, Rukmini Devi Institute of Advanced Studies,GGSIPU, Delhi

${ }^{3}$ Ms.AmandeepKaur, Assistant Professor, Rukmini Devi Institute of Advanced Studies,GGSIPU, Delhi

${ }^{4}$ Ms.AnshuLochab, Assistant Professor, Rukmini Devi Institute of Advanced Studies,GGSIPU, Delhi

Abstract:Purpose:Childrenarethefutureofev erysociety. Thewelfareanddevelopmentofchil d'sphysical,mentalandemotional phase are very essential for the growth of children into mature and intelligent adults. Child labor is a significant problem in India. The present research study is an attempt to provide a strategic framework for eradication of child labor child labor engaged in Dhabas of Delhi.

Design/ Methodology/Approach:The primary data on current study was collected on the basis of Convenience sampling method from 150 child laborers in all four zones in Delhi through questionnaire survey. Chi-

squaretesthasbeenusedtofindoutthefactorssig nificantlyassociatedwiththelevelofchildlabor. Originality/Value: The researchers have created an original database with the help of 150 childlaborers of Delhi. The research team has conducted an original study that has attained a workable and truthful conclusion. The results of the study provides a strategic framework for eradication of child labor child labor engaged in Dhabas of Delhi.

Key words: -Child Labor,Dhabas, Poverty, Livingconditions, exploitation 


\section{Introduction:-}

ChildrenarethesupremegiftontheearthandtheyaretherepresentationofthebeautifulcreationofGod.Th erichnessandprogressofanycountrywouldcertainlydependsupon

howdevelopmentorthewellbeingofitspeoplein

generalandchildreninparticular, thanthedevelopmentoftheirmilitaryoreconomicassetorthebrilliance oftheircapitalcitiesandpublicbuildings.

Sincecenturies, childlaborhasbeenfundamentalacrosstheworldanditisauniversalproblem. Thispracti ceinanysocietyisviolationofhumanrightsasitisbrutal,irrational, inhuman\&degradingtheethosofthech ildren.Thusthechildlaborisnotonlya social evilbut it isadisguiseonthechildhood\&exploitationofthechildren.

Thechild

laborisacontroversialtermtodefine,especiallyinthecontextofchildlabour.ChildworkandChildlabour oftenusedsynonymouslyanditisbelievedthatallworkisnotbadforchildrenbecausesomelightwork, pro perlystructuredandregulated,isnotqualifiedaschildlabour.Thisimplies

thatworkwhichdoesnotinterfereotheressentialactivitiesofchildrensuchasleisure, playandeducationar enotchildlabor. Childlabor,therefore, istheworkwhichinvolvesevenminimumlevelofexploitationnam ely,physical,mental,economicandsocialandtherefore,impairsthehealthanddevelopmentofchildren.I tispointlesstotryanddistinguishbetweenchildlabourandchildworkorbetweenhazardousandnonhazardousemployment. Workthatseemstobenon-

hazardousforadultsbecomeshazardousforchildrenbecausetheyhavenonegotiatingpower.Withregard totheproblemrelatedtoconceptanddefinition, therearetwoschoolsofthought.

Accordingtothefirstschoolknownasabolitionistschool,educationshouldbemadeafundamentalhu manrightforevery childin514agegroups, andanychildwhoisoutofschoolshouldbetreatedasaimpendingworkingchild. Theyfeelth atabolitionofchildlabourandattainmentofcompulsoryprimaryeducationaretwosidesofthesamecoina ndonecannotbeachievedwithoutachievingtheother.Accordingtothem, thedistinctionbetweenhazard ousandnon-hazardousworkisnotimportant.

Accordingtothesecondschoolknownasreformistschool,child

laborisaharshreality, ,reflectingthesocio-

economicconditionsofIndia(likepoverty,unemploymentandilliteracy)itisimpossibletogetridofthepr oblemofchildlabouraltogether.Theyfeelthateliminationofchildlabourshouldbeviewedasalongtermgoaltobeachievedprogressively.Hence,theyadvocateadualapproachof prohibitionof child labourin hazardous workandregulationofitin non-hazardouswork.

\section{Various definitionsofChild Labor:}

AccordingtoUNConventionontheRightsofChild,"Childlabourinvolveseconomicexploitationandp erformanceofworkthatishazardousorinterfereswiththechild'seducation, orisharmfultothechild'shealt horphysical,mental,spiritual,moralandsocialdevelopment."Anorganizationconcentratingonchildwo rkersdescribedchildlabouras,"Apersonwhohasnotcompletedhis/her15yearsofageandwhoisworking withorwithoutwages/incomeeitheronaparttimeorfulltimebasis."5

AccordingtoInternationalLabourOrganisation(ILO),"Childlabourincludeschildrenpermanently leadingadultlives, workinglonghoursforlowwagesunderconditionsdamagingtotheirhealthandtotheir physicalandmentaldevelopment.Sometimesseparatedfromtheirfamilies, frequentlydeprivedofmean ingfuleducationandtrainingopportunitiesthatcouldopenupforthemabetterfuture."asTheConstitution oflndiadefinesachildasonewhoislessthan14yearsofage.However,theInternationalLabourOrganisati onhassetforthanagelimitoffifteenyearstodefine achild.Insomecountrieschildrenare defined as thosewho 
arebeloweighteenyearsofage. Thus, theminimumagelimittodefineachildinIndiaiscomparativelylowe rthaninternationallyapprovedage-limit.

Asaresult,therearevariousestimatesforthecalculationofnumbersofchildlabourersinIndia.Goingbyth efiguresoftheInternationalLabourOrganisation(ILO), itISestimatedthatmore

than73Millionchildrenareeconomicallyactivein 1995."ThegreatestnumberswerefoundinAsia,44.6m illion(13percent),followedbyAfrica,

23.6million(byfarthehighestrateat26.6percent), andLatinAmerica,5.1 million(9.8percent).

\section{Child Labor in India:VariousPerspectives ontheProblem}

Childlaborisoneofthemostimportantandurgentproblemsthatneedsattentiontoday. Itisthebaneforanyso cietyandhasanimpactthatfaroutweighstherelatedproblemstheycause.Theyhavethepotentialtoimped ethegrowthofsocietyandhaveadominanteffectonmanyproblemsinthesociety.Itisaproblemthatnotonl yaffectsthechildworkersandtheirdevelopment, butalsothedevelopmentofthesociety. Oneneednotign orethe fact

thatchildren arethefutureofoursociety.Thewelfareanddevelopmentofchild'sphysical,mentalandemotionalfaculti esareveryessentialforthegrowthtoturnthemintomatureandintelligentadults. Thechildrenarethetorchb earersofthefutureandtheirgrowthtomakethemresponsiblemembersofthesocietyisessentialforthedev elopmentofanysociety.Childlabourwithitsvariousproblemsofilliteracy,health,antisocialactivitiesandlackofdevelopmentofchildren-bothphysicallyandmentallyposesaseriouschallengeforsocietalsynchronizationanddevelopment.Beforeproceedingfurtheraclarif icationoftheconceptofchildlabor isnecessary.

Childlaborisnotanewphenomenonhuthasbeenpracticedfromancienttimes.InancientIndia,childlabor isfoundintheformofchildslavery.Childrenbeloweightyearswereownedbyrichpeopletordoingmenial jobsduringtheperiodofChandraguptaMaurya. Hismentor alsomentions inhis 'Arthasastra'aboutthis ignoblepractice. ThispracticetookanewforminmedievalIndia.Duringthisperiod, aclassoflandlesslab orersemerged.Theselaborersusedtheirchildrentohelpintheireconomicactivities. Theperiodalsowitne ssedtheadventofcraftandtradeandthecraftsmenalwaysusedtheservicesofthechildreninthefamily. Wit hoccupationsbeingdecidedonthebasisofheredity,thechildrenwereexposedtothetraditionalcraftatane arlyage.UndertheMughals, wealsolendthechildrenbeingusedasslaves. Withtheemergenceofindustria lization, duringthemodernperiod,thechildrenwere,forthefirsttime,removedfromtheirdomesticsurrou ndingsandtheirlabourwasexploitedforcommercialgains; Childrenwereforcedtoworktorlonghoursan dunderhazardousconditions. ThiscontinuedintoindependentIndiaandthemalaisehasbeenwidespread evenafterindependence.

\section{REVIEW OF LITERATURE:}

Indiacontinuestohostthelargestnumberofchildlabourersintheworldtoday.AccordingtotheCensus 20 01, therewere 12.7millioneconomicallyactivechildrenintheage-groupof5-

14years. Thenumberwas11.3millionduring1991(PopulationCensus)thusshowinganincreaseinthenu mberofchildlabourers. Workersingeneralareclassifiedintomainandmarginalworkers 1 bythepopulati oncensus.Censusdatashowsthatthereisadeclineintheabsolutenumberaswellthepercentageofchildre $\mathrm{n}(5-$

14)tototalpopulationinthatagegroup,classifiedasmainworkersfrom 4.3 percentin1991 to 2.3 percentin 2001.Buttherewasasubstantialincreaseinmarginalworkersineverycategoryofworkerirrespectiveofs exandresidence.Asaresult,despitethenumberofmainworkersdecliningfrom9.08millionin1991 to5.7 8millionin2001, thetotalnumberofchildrenintheworkforceincreased.Alargepartoftheincreasewasac countedforbytheincreaseinmarginalworkers, whichincreasedfrom 2.2 million in 1991 to 6.89 million in 2001. Thetrends between1991 and 2001ofdecliningmainchildworkersalongwithincreasingmarginalworkersmayindicatethechangingn atureofworkdonebychildren. Thisisalsotobeseeninthecontextofdeceleratingemploymentgrowth 
ingeneral in theeconomyduringthe last decade.

TheFederalLaborActGovernmenthavesettheminimumagefortheemploymentofchildrenattwelveyea rsandisinforceinallthe36statesofNigeria.TheNigeria"sLaborAct permits childrenatanyage to performlightwork in domestic service orworkwithfamilymemberinagriculture.However,theChildRightsActprohibitstheworstformsofchil dlabour,includingtheforcedlabourofchildrenanduseofchildreninprostitutionorinarmedconflict.The LaborActsetsdifferentagesforvarioushazardousoccupations. Forinstance, achildagedfifteenorolderc anworkinindustries. Thelaw forbidschildrenunderageofsixteentoworkundergroundortoworkwithma chinesbutclearlyallowchildrenagedbetweensixteentoeighteentodothesehazardousoccupations. How ever,thesamelawprohibitsemploymentofchildundertheageofEighteentoworkinharmfulenvironmen t.Thelawdoesnotremovechildrenfromdomesticservice, thiscanincreasedincentivesforparentstosend theirchildren to work (United StatesDepartment ofLabor, 2011).

In2002NigeriaratifiedConventionNo.138,theMinimumAgeConventionandConventionNo.182, the WorstFormsofChildLabour.NigeriaalsoadoptedtheUnitedNationsConventionontheRightsoftheChi ld, andthe AfricanCharterontheRightsandWelfareoftheChild, whichappearedtohavelaidresttothearg umentthatchildrenhavenoclearlydefinablerightsinNigeria.BoththeConventionontheRightsoftheChi ldandtheConventionontheEliminationofallFormsofDiscriminationagainstWomen(CEDAW)werea doptedin1991 and1985respectively.HoweverboththeCRCandCEDAWhavenowbeen"domesticated "inNigeria.TheAfricanCharterhasalsobeendomesticatedbyNigeria.However,theNationalAssembly shouldseriouslylookintotheseandotherinternationallaws, especiallyhumanrightsissuesthatadversely affect therights and fundamental freedoms of thecitizenry. Thus, the problemnow is how effectivelytoenforce and monitor theimplementation of theseprovisionsastheyaffectchildren ${ }^{e c}$ sandwomen ${ }^{e e}$ srightsinNigeria.Thisalsopresupposesthatallsoci alrightsshouldbemadejustifiableinNigeriasoastoempowerthelessprivilegedin thesociety(Onyemachi, 2010).

TheNigeriangovernmenthasprovidedanenablingenvironmentandsupportforthesecivilsocietyorgani zations(CSOs)tothriveandhasdrawnfromtheirworkformulatepolicies, programmesandinterventions forchildvictimsofabuseandviolence(Ekpenyong\&Sibirii,2011).On2000, theNigerianGovernmentes tablishedanationalprogramtoeliminatetheworstformsofchildlabourinNigeria(Elijah\&Okoruwa,200 6).Despiteallthesechildrenarestillabused.EkpenyongandSibirii(2011)statesthereasonchildlabouris prevalent duetotheeconomicsituationwheremanyfamilieslivebelowpovertyandcanbarelyearnenoughtofeedth emselvesandtheirchildren.Furthermoremainlychildlaborersareengagedatthehouseholdlevelorstreet hawkingTogundeandArielle(2008)arguesthatregulationsregardingstreethawkinghave been difficult to control bythegovernment.

\section{RESEARCH METHODOLOGY:}

\section{Significance of the Study:}

The concernfortheeradicationofchildlabourhasbecomeamajorsocialagendatowhichbothcivilsocietyand statepayagreatdealorattentiontodaytorootitoutfromthefaceofthenation.

\section{Scope for FutureStudy:}

The futureresearchersshouldtargetNCRdhabasinordertohavestrongresultssincedhabasonhighwaysconst itutemajorportions of childlabor.

\section{Objectives of the Study:}


- To measurethe magnitudeof child laborworkingin Dhabas at Delhi.

- Tounderstandthecompositionofchildlabor(A)SocialComposition:religionwise,casewise,regionwis e,andagewise.(B)EconomicComposition:OccupationandIncomecomposition of the parents.

- Impact of migrationonchildlabor.

- To analyze the causes of childlabor: Social V/s Economic causes.

- To study the impact of childlabor on childrenpsychology,their health, theireducationand theirfamilylife etc.

- To develop a model for theeradication of childlaborin Delhi

\section{Research Design:}

Thepresentresearchworkisdescriptiveandanalogicalthatincludessurveysandfactfindingenquiriesreg ardingexistingliteratureandevents.

\section{Data Collection Method:}

Inordertoaccomplishtheobjectivesofthestudy,combinationofbothqualitativeandquantitativeresearc hmethodshavebeenadopted.The designofthe studyisdescriptive.TheNonProbabilitysamplingmethodhasbeenusedtodrawtherequiredsampleofchildlaborers.Besidestheseres pondents, thequalitativedatawascollectedusingnarrativeanalysisasamethodofdatacollection. The datahasbeencollectedusingself-

administeredinterviewschedulewiththechildlaborers. Thesecondarysourcesweretappedandinformat ionprocuredfromthemwasanalyzed.Thiswasprimarilypertainingtotheconstitutionalprovisions, vari ouslegislations, censusdata,ILOreports, andrelevantreportsofMinistryofLabor, GovernmentofIndia, ReportsofGovernmentofDelhi,Books,Journals,PeriodicalsandReportsofNongovernmentalorganizations.

Selection

of

Sample: Mostofthestudieshavebeenconductedinalreadyidentifiedindustries, butnotmuchattentionhasbeengi ventothechildlaborworkinginaroadside Dhabas. Therefore, for the current study, sample has been taken form roadside Dhabas of Delhi.

Area of Sample: The study was conducted in all four zones in Delhi.

Sample Size: The sample targeted for survey was 150 child laborers in Delhi in all four zones; East, North, West and South. However, we received 100 completely filled accurate questionnaires.

Choice of Respondents:Convenience sampling method has been followed in choosing a sample of 150 child labourers working in dhabas situated in Delhi. Chi- square test has been used to find out the factors significantly associated with the level of child labor

Design of Questionnaire: Datawascollectedthroughselfstructuredquestionnaire.Thedatacollectedwerethentabulatedandanalyzedforthepurpose.Variousasp ectscoveredin the questionnairearelistedbelow:Profile of thechildlaborer, Familybackground, Livingconditions- Healthandhygiene concerns, Workingcondition and Financialconcerns

Testing of Questionnaire: Thestructuredquestionnairewastestedthroughafocusgroupinterviewamongthe5collegestudentswhe retheywereinterviewedoneachparameterdraftedandthemoderatorobservedthatallthequestionswerer elevantexceptfewi.e.,3questionswhichwerenotvalidincontextofDelhiregion.Outof41questionsorigi nallyframed,3 weredropped andfinallythe pilot studycarriedon with 38 questions. 
Period of Study: Thepresentstudycoversaperiodof15yearsbeginningfrom2000-

2015forsecondarydataand April, 2015 - June, 2015 forthecollection of primarydata

DataAnalysisMethod :The method to be used is Bivariate analysis-tests of differences-t test for comparing two means and ANOVA for complex experimental designs. Also to test goodness of fit and independence, chi-square test will be used.

\section{Statistical Tools andTests Used for Testing ofHypothesis :}

SPSSstatistics 21 softwarewould beusedforthefollowingtests:

$\checkmark$ Descriptive statistics

$\checkmark$ ParametricTest-T- test,ANOVA

$\checkmark$ Non parametricTest- Mann Whitney,Kruskal-Walis, Chai-square test.

\section{RESULT \& FINDINGS:}

Ofthe respondents (06\%) surveyedwere femaleand (93\%) weremale.

Table1:Demographic Variable

\begin{tabular}{|l|l|l|}
\hline Details & Frequency(N) & Percentage \\
\hline Gender: Male & 76 & $\begin{array}{l}93 \% \\
6 \%\end{array}$ \\
\hline Female & 5 & $27 \%$ \\
$10-14$ & 22 & $73 \%$ \\
\hline MaritalStatus: Married & 59 & $1 \%$ \\
Unmarried & 1 & $99 \%$ \\
\hline
\end{tabular}

\section{Cronbach alpha test- Test for reliabilityscale}

ReliabilityMeasures:ReliabilitytestwascarriedoutusingSPSSSoftwareandthetestmeasur esaregivenbelowinTable2below.FaceValidityof questionnaireswastestedand it was found to be satisfactory.

Table2 :ReliabilityStatistics

\begin{tabular}{|c|c|c|}
\hline Cronbach'sAlpha & $\begin{array}{c}\text { Cronbach'sAlpha } \\
\text { BasedonStandardizedItems }\end{array}$ & Nof Items \\
\hline .70 & .70 & 38 \\
\hline
\end{tabular}

Table 3: Distribution of Child Labor on Age and Gender Bases

\begin{tabular}{|l|l|l|l|l|}
\hline \multirow{4}{*}{ Age } & \multicolumn{3}{|c|}{ Gender } & \\
\hline \multirow{4}{*}{} & Male & Female & Total \\
\cline { 2 - 6 } & $6<=$ & 4 & 0 & 4 \\
\cline { 2 - 6 } & $8-10$ & 15 & 3 & 18 \\
\cline { 2 - 5 } & $10-12$ & 46 & 4 & 50 \\
\cline { 2 - 5 } & $12-14$ & 28 & 0 & 28 \\
\hline
\end{tabular}


Table 4: Distribution of Child Labor on Proportion on Literacy Basis

\begin{tabular}{|c|l|l|l|l|l|}
\hline Education & \multicolumn{5}{|c|}{ Age } \\
\hline & $6-8$ & $8-10$ & $10-12$ & $12-14$ & Total \\
\hline Not Enrolled & 0 & 0 & 6 & 0 & 6 \\
\hline Dropped out & 4 & 14 & 25 & 24 & 67 \\
\hline Attending & 0 & 4 & 1 & 8 & 13 \\
\hline Complete & 0 & 0 & 0 & 14 & 14 \\
\hline Total & 4 & 18 & 32 & 46 & 100 \\
\hline
\end{tabular}

Table 5: Pearson's Chi-Square Test on Child Labor on Proportion on Literacy Basis

\begin{tabular}{|c|l|l|r|}
\hline & Value & df & Asymp. Sig. (2-sided) \\
\hline Pearson Chi - Square & $37.028^{\mathrm{a}}$ & 9 & .000 \\
\hline Likelihood Ratio & 44.566 & 9 & .000 \\
\hline Linear-by-Linear Association & 12.974 & 1 & .000 \\
\hline N of Valid Cases & 100 & & \\
\hline
\end{tabular}

Interpretation: 11 cells $(68.8 \%)$ have expected count less than 5 . The minimum expected count is .24. Chi square value at 9 degree of freedom is 37.028 and its $p$ value is 0.00 which is less than 0.05 , so it may be concluded that the proportion does not significantly differ in the given category of the given literacy which clearely reflects that the intensity of child labour is more in case of drop out in case of literacy which is a critical issue for policy makers.

\section{Gender VsMonthlyEarnings}

H0:ThereisnosignificantdifferencebetweenmaleandfemalemonthlyearningsinDhaba

$\mathrm{H} 1$ :ThereisasignificantdifferencebetweenmaleandfemalemonthlyearningsinDhaba

\section{T- Test:Test of SignificanceBetweenMonthly Earnings\& Gender of TheRespondents}

Table 6: Group Statistics

\begin{tabular}{|l|l|r|r|l|c|}
\hline & Gender & $\mathrm{N}$ & Mean & $\begin{array}{l}\text { Std. } \\
\text { Deviation }\end{array}$ & Std. ErrorMean \\
\hline \multirow{2}{*}{ Monthly Earning } & Male & 76 & 3.2700 & .53617 & .11989 \\
& & 5 & 3.0500 & .81531 & .18231 \\
\hline
\end{tabular}

Table7 :IndependentSamplesTest

\begin{tabular}{|c|c|l|l|l|}
\hline $\begin{array}{c}\text { Levene'sTestforEquality } \\
\text { of } \\
\text { Variances }\end{array}$ & \multicolumn{2}{l|}{$\begin{array}{l}\text { T- } \\
\text { TestforEqualityof } \\
\text { Means }\end{array}$} \\
\hline F & Sig. & t & df & $\begin{array}{l}\text { S } \\
\text { i } \\
\text { g }\end{array}$ \\
\hline
\end{tabular}




\begin{tabular}{|c|c|c|c|c|}
\hline $\begin{array}{l}\text { Equalvariances } \\
\text { Monthly Earningassumed } \\
\text { Equalvariances notassumed }\end{array}$ & $\begin{array}{l}1 . \\
4 \\
7\end{array}$ & .232 & $\begin{array}{l}1.0 \\
08 \\
1.0 \\
08\end{array}$ & $\begin{array}{r}38 \\
32.844\end{array}$ \\
\hline
\end{tabular}

TheabovetableindicatethatSignificantvalueis0.320whichisgreaterthan $(\mathrm{P}>0.05)$, hencethenullhypo thesisisacceptedandwemayconcludethatthereisnosignificantdifference between maleandfemale monthlyearnings in Dhabas. 
TableNo. 8Occupational structure ofthe Family

\begin{tabular}{|l|r|r|}
\hline Occupation & Mother & Father \\
\hline Labourer & 37 & 62 \\
\hline Vendor & 3 & 2 \\
\hline Mechnaic & 0 & 2 \\
\hline Rickshaw Puller & 0 & 12 \\
\hline Dead & 20 & 12 \\
\hline Housework & 40 & 10 \\
\hline
\end{tabular}

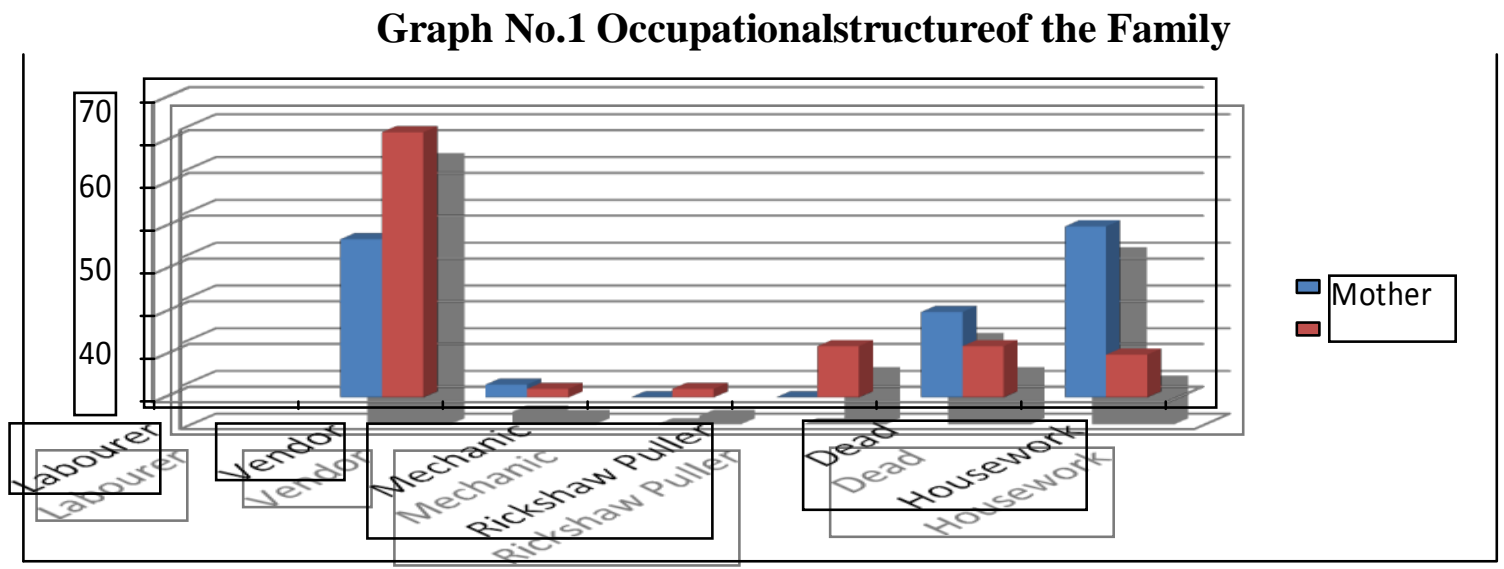

\section{FINDINGS:}

Thefindings areasfollows:

- Findingsrelated to identificationData:

$>$ Itisstatedthat,theproportionsofmalechildlabourworkingindhabasaremore(93\%)thanfemal es ${ }^{\text {ee }}$ childlabour(07\%).Manyofthechild

labourareworkingindhabasforsupportingtheirfamilyincomeasthechildhas to sharethe responsibility.29respondentsshared that theyhavetotakecareoftheirfamilyand13hadlosttheirfathers/mothersorboth.Thus,42respon dentsaretakingfullresponsibilityoftheirfamilyandrestwasforcedtoworktofulfilltheirparent ${ }^{\text {ee }}$ swish.

Thechildlabourersworkingindhabaswantstotakeeducationandwanttogotoschool,howevero nly20percentwerefoundtobein1-5standardand9percentin6-

8standardandrestwerehavingnoeducationatall.It isobserved that,mostof the child laborerscouldnetable toattaintheir education becauseoftheirregularlabour jobsin dhabas of Delhi

> ItwasalsoobservedthatmostofthechildlaborbelongstoBiharandBengalandleast from Jharkhandandremoteareas of UttarPradesh.

- Findingsrelated toFamilyFactors:

$>$ Inthepresentstudy, mostofthechildlaborersarefromthejointfamiliesandthey haveanaveragefamilysizeof5persons.ChildlaborersusuallygotfamilybrokenofDivorce, mar italconflicts, parent ${ }^{\text {ee }}$ salcoholicaddiction and death of their parents.

- Mostofthechildlabor sparentsdonotworkwiththematthesameplace.93\%oftherespondentsr 
eportedthattheirparents, fathersworkinconstructionsandmothersashousemaidsorbothincon struction.However,in7\% casesitwasobservedthattheirfatherormotherworkin thesamedhabaas a headcleaner or ahelper.

$>$ Itwasnotstrangetonoticethatthatnoneofthemwereasinglechildinthefamily. Allthechildlabor ershavetheirsiblingsandtheyarealsoworkingin differentplaces.

- Findingsrelated toLivingConditions:

> Whenaskedrespondentsabouttheirnativeplaceandhomes, theyreportedinmuchelucidatedm annerwhereitwasfoundthatallofthemwerefromremoteareasor slums of rural livinginthatchedhouse.

$>$ Thereweredifferentreasonsforthemtoleavetheirnativeplacesbutthemajorproblemwhichwa sunderstoodwaspovertyindeed,however, tosurvivetheliving,theproblemsfacedwerereporte dasapproximate $60 \%$ ofthechildrenreportednosourceofemploymentforthewholefamilyandn oextrafacilitiesprovidedwitheducationand20\% reportedthatnomedicalfacilitiesavailablefor chronicdiseasesandrest $20 \%$ childrenreportedthat heydidnotwanttoconfinetooneplaceandra therwantedto explorethe opportunities outside their villages.

$>$ Whenaskedaboutthelivingconditions, allofthemagreedthatallthefactorsspecificallyhealtha ndhygienearebetterinDelhithanattheirown native places.

- Findingsrelated toOccupationalData:

$>$ Itwasfoundthat $70 \%$ ofthechildrenwereengagedinthesamedhabaforayear,howeverresthave beennewtothisplaceandfoundtoworkinginotherareaofemployment. $\%$ childrenmentioned thattheyweregoingtoschoolintheirnativeplacebutparentsaskedthemtoleavetheirschoolsan dstart workingastheycould not afford the education.

- Findingsrelated to Working Conditions:

$>$ The childlaborsindhabasaremajorlyresponsibleforcleanertypework,typically

workingfor5-6hoursonashiftbasisas60\%childrenwerefoundto be studying while workingeither in morningor eveningshift.

$>$ Whenaskedaboutearnings, 20\%childrendid not knowabouttheirearningsastheirparentsareentitletogettheseandrestsaid, theyearnsomewher earoundRs.300-

600permonthwithmealsfree. Therearefoureconomicfactorsfoundinthepresentstudy, sucha slowwages, nowagesforovertime,FringeBenefits, delayinpaymentofwages \&EmployerEmployeeRelationship.Childlaborersaretypically paidlessthanadultsinallvarietiesofjobseventhoughtheyperformthesameworkand haveto workbeyond normal workinghours.

$>$ Thedistributionoftherespondentsaccordingtothedelayedpaymentoftheirwages, thechildla borersrevealsthat, theyhadalwaysdelayedinpayingtheirwagespaymentwithoutfixingthedat eofpayment,whichiswithout "Payment of wagesAct" for adults.

$>$ Noneof thechildrenreported anycaseof punishment byanemployee.

$>$ Most ofthechildrendid not knowiftheyweresufferingfromanydisease but $30 \%$ reportedoccasionallyfever,cough, andcolddueto dust,dirtywaterandexertion. Theyalsomentionedthattheygetpaiddayoffand visit Govt. hospital havingno orlessfess forOPD.

$>$ Inallthecases, thechildrenweregetting2mealsinadayand $30 \%$ ofthe childrenalsogot onepair ofdress foranyearwhichtheywash onceaweek.

$>$ ThechildrenreportedthattheyoftengettipswhilecleaningthedeskandtheyearnaroundRs.200 permonthfromtipsfromwhichtheybuynecessities fortheir family. 
- OtherFactorsCausesof Child Labour

$>$ Socio-

economicfactorsareprimarilyresponsibleforperpetuationofchildlabourinDelhi. Widesprea dandchroniceconomicpovertyintheurban\&ruralareasforcesparentstosendtheirchildrenfor work; casteism,genderdiscrimination, largefamilysize, lowlevelofincome,geographicallyd isadvantagedarea,illiteracyetc.docontributesignificantlytothecauseofchildlabourpractice. Furtherwithadvantageslikelowremuneration,lackofunions, ondemandforovertime,simple andeasytobemoldedandpunishedetc.,beinginherentwithchildren,employerspreferchildlab our.Inmostunderdevelopedandagrariansocietychildrenarethepartoflabourforce. Withthegr owthofurbanizationandindustrialization, theyaremovingfromfamiliesto hotel and otheremployment.

$>$ Unsafeandunregulatedworkingconditionsaffectthephysicalandpsychologicalhealth of the labour.

\section{RECOMMENDATION:}

Theprevalenceofchild

laborisshown bythechildworkparticipationrates, whicharehigherinIndiathaninotherdevelopingcountries. Thema jordeterminantofchildlabourispoverty.Eventhoughchildrenarepaidlessthan adults, whatever income theyearn is ofbenefit to poorfamilies.

Changingthestructureoftheworkforceandhiringahighnumberofcurrentlyunemployedadultsingrea tlyimprovedworkconditionsisonlythefirststepinthislengthyprocess. Acycleofpovertyisformedand theneedforchildlabourisrebornaftereverygeneration.Indianeedstoaddressthesituationbytacklingt heunderlying

causesofchildlabourthroughgovernmentalpoliciesandtheenforcementofthesepolicies. Onlythen willIndiasucceed inthe fightagainstchildlabor.

Thefollowing arethe recommendationsaccording to thestudyconducted:

- Thereshouldbecompulsoryeducationtoallschoolagedchildrenbecauseeducationhelpschild ren learn skills that will helpthem earna living.

- Theage ofchildren should be amendedfrom 14years to 16years.

- Childrenshouldbepaidthesamewageasadults;thiswillremovetheeconomicin-centiveto employchildlabourers.

- Childrenshouldbeprovidedwithnonformaljoborientededucation,suchthatitenhancestheirskill,whichwouldenablethemtoshiftt omoreremunerativesatisfactionand less hazardous alternativeemployment.

- Thereshouldbeapracticaltimeboundstandardizationoruniformityprogramsto eliminatechild labour

- Thelegislatureshoulddefine,,childlabor ${ }^{\text {ee }}$ andtherebyremovetheconfusionregardingthe scopeofchild labour

- Theconstitution should cast a dutyon theguardian to send the child to school.

- Not only governmentbut alsoevery citizentakesto responsibilityto givefinancial support to everyeconomicallypoorfamily

\section{CONCLUSION:}

Thechildrenaretheleadersofthefutureandtheirdevelopmentintoresponsiblemembersofthesocietyis vitaltothedevelopmentofanysociety.Childrenconstituteabout40percentofthetotalpopulationofIndi a.TheconstitutionofIndiamentionsachildasonewhoislessthan14yearsofage.Developmentofachildi 
nallitsareas-physical,cognitive, social,andemotionalgrowsatarapidrateduringtheearlyyears. AccordingtoIndiannominculture, humanlifeconsistsoffour uniquephasesviz.,childhood(Balyavastha), adulthood(Kumaravastha),youth(Yavvanavastha)ando ld

age(Vardhakyavastha).Childhoodplaysanimperativeroleinone es slifebecausethisistheonlyphasewh ichunfoldstheabilitiesandskillshiddeninsidethechildandpavesthewayfordevelopmenttothefullexte nt.

Indhabachildren, duetounfavorableconditions, whichincludelivingandworkinginunhealthyenviro nment,lackofcareandaffectionofparents, lackofhealthyinteractionwiththeoutsideworldhamperthe developmentofthechild.Insteadofattendingschoolanddoingotheractivitiesnormaltotheirage,these childrenareforcedtoplaytheroleof anadult.Thesechildrenhave no scopetosocializeintotheirnormalroles. Thesechildreneasilyfallpreytoantisocialactivitiesand.thereisagreatdangerofthembecomingpotentialcriminals.

\section{REFERENCES:}

- $\quad$ Census ofIndia 2014. Series-1,India,ProvisionalPopulation Totals,Paper 1 of2001. NewDelhi:Officeofthe RegistrarGeneral

- Gangrade,KD, Gandhi JA 1983. Women and Child Workers in UnorganisedSector.NewDelhi:Concept PublishingCompany.

- $\quad$ GeorgeI1990. ChildLabour and Child Work. NewDelhi:AshishPublishingHouse.

- ILO1983. Report oftheDirectorGeneral.Thirteenth

- InternationalConferenceofLabour Statisticians.Geneva:InternationalLabourOffice.

- Khan MA 1979. SociologicalAspects of Child Development.NewDelhi:Concept PublishingCompany.

- Mishra L2000. ChildLabourinIndia.NewDelhi: Oxford UniversityPress.

- Singh M 1980. Working Children in Bombay:A Study. New Delhi:ShipraPublications.

- Suman CK 1998. Problems and issues on child labourinIndia.Social Action, 48:19-34.

- WeinerM 1991. TheChild and the StateinIndia.NewDelhi: Oxford UniversityPress.

- ZutshiB, Joshi S, Vajpeyl A 2006. Review of ChildLabour,EducationandPovertyAgenda.India CountryReport. New Delhi: GlobalMarchAgainstChildLabour.

- B.R.Patil,Eliminating Child Labour:Some National andInternationalInitiatives,Ibid., p.172

- DevakiJainandMaliniChand,"RuralChildrenatWork:PreliminaryResultsofaPilot Study...IndianJournal c?(Soc:ialWork,Vol.XL, No.3,Oct. 1979

- HelenR.Sekar,ChildLabourLegislationinIndia:AStudyinRetmspcctamll'mspect,Natio nalResourceCentreonChildLabour,V.V.GiriNationalLabourInstitute, Noida(U.P), 1997, p.7. 
- India ChildLabour,NSSO, 2006.

- Indian Penal Code, Section 82

- I.S.Singh, ChildLabour,Delhi,OxfordIBH PublishingCo. Pvt.Ltd .. 1992.

- K. SumanChandra,ProblemsandIssueson Child Labourin India,SocialChange,Vol.27, Sept-Dec. 1997, p.34.

- Myron Weiner, TheChild and the State inIndia,Delhi: Oxford UniversityPress.1991, p.3.

- NationalResourceCentreonChildLabour,V.V.GiriNationalLabourInstitute,

- Noida (U.P), 1997, p.7.

- P.L.Mehta, ChildLabourandLaw,Delhi:Deep\&DeepPublications, 1996

- SatishDevi,ChildWorkersinHaryana,Soc:ialll'e(fure,Vol.XXVI.No.7.Ocl.1979

- V.Verma. ChildLabour-Need for Social Awareness, Yojana,23(2) 13-14,197. 
